\title{
Unsupervised segmentation of stents corrupted by artifacts in medical X-ray images
}

\author{
Hugo Gangloff \\ ICube, Université de Strasbourg \\ CNRS UMR 7357 \\ Illkirch, FRANCE \\ GEPROVAS \\ Strasbourg, FRANCE \\ hugogangloff@unistra.fr \\ Nabil Chakfé \\ Department of Vascular Surgery \\ and Kidney Transplantation, CHRU \\ Strasbourg, FRANCE \\ GEPROVAS \\ Strasbourg, FRANCE \\ nabil.chakfe@chru-strasbourg.fr
}

\author{
Emmanuel Monfrini \\ SAMOVAR \\ Télécom SudParis \\ Institut Polytechnique de Paris \\ Évry, FRANCE \\ emmanuel.monfrini@telecom-sudparis.eu
}

\author{
Christophe Collet \\ ICube, Université de Strasbourg \\ CNRS UMR 7357 \\ Illkirch, FRANCE \\ c.collet@unistra.fr
}

\begin{abstract}
We propose a new methodology for the segmentation of stents in 3D X-ray acquisitions. Such data are often corrupted by strong artifacts around the stent, requiring the development of a robust algorithm: because of the medical application, we need to produce an accurate segmentation. Moreover, we aim at developping a robust technique that can handle heterogeneous data. We propose a two-step, coarse-to-fine approach, that handles the corrupted cases. This approach leads to better results illustrated in the context of metallic artefact reduction.

Index Terms-image segmentation, probabilistic and statistical models, metal artifacts, X-ray imaging
\end{abstract}

\section{INTRODUCTION}

Stent has become a standard treatement to cardiovascular diseases. Yet, its in vivo behaviour is far from being fully understood [11]. The amount of medical images is constantly growing offering new opportunities to learn from clinical cases. The interactions between the stent struts and the calcifications are believed to be a major cause of failure of the treatments based on the implantation of a stent. The study of such interactions by combining information of both $\mathrm{CT}$ and micro-CT (mCT) scans ${ }^{1}$ is a new approach to the problem and requires the development of new imaging tools able to finely segment the metallic stent components.

The most complex images to process often include a broken stent in a calcified environnement. The image is also often

The authors would like to thank association GEPROVAS for the funding of this work (https://geprovas.org).

${ }^{1} \mathrm{CT}$ scan images are the clinical standard data while mCT scan images are acquired during studies subsequent to the explantation of the stented artery. The latter offer a much better spatial resolution. corrupted by strong artifacts around the stent which originate from physical interactions between X-rays and the metallic stent. To the best of our knowledge, there exists no dedicated method to confidently segment stents in such images in an unsupervised fashion. As a consequence, people still use simple approaches such as manual thresholding which fails in complex cases. Thus, such cases are discarded from clinical data sets despite their medical interest [16].

\section{A. Our experimental data}

Our images consist of X-ray scans (CT and $\mathrm{mCT}$ ) of stents from the Geprovas database [11]. Our data thus consist of cubes of grayscaled voxels. Such a dataset is challenging because of the heterogeneity of the data linked with the stent model, the presence of calcifications, the stent deterioration, the device used for image acquisition and the wide variety of failure causes. Some slices of these data cubes are visible in Figure 1, where the stent artifacts are notable.

\section{B. Stent segmentation in medical data}

We now give a brief overview of the work achieved by other researchers to perform stent segmentation in medical images. Relatively few works deal directly with the topic.

Works such as [9] or [10] that also address stent segmentation are not suitable for us since they do not consider the stent in a calcified environnement. Calcifications and stent are so close in appearance and sometimes in geometry that dedicated methods need to be developped to distinguish both classes during segmentation. Moreover, we have to discard approaches such as [5] since they are stent specific and we need our method to be independent on the stent model to process our data. 

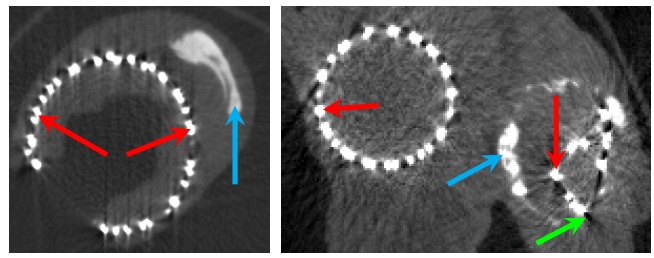

(a) mCT scan

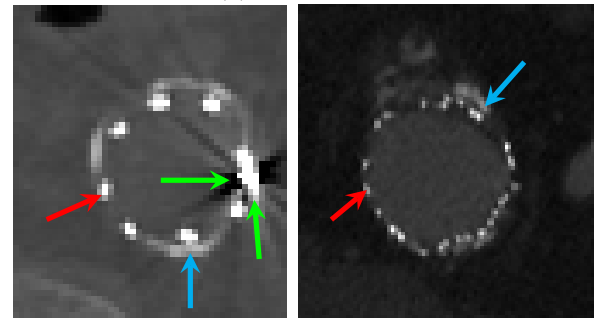

(b) CT scan

Fig. 1: Example of input data (2D views), we see the complex environnement in which the stent lies: artifacts and calcifications are notable. Some stent, calcification and artifact components are indicated by, respectively, red, blue and green arrows. We note the spatial resolution differences between CT scans and mCT scans.

However, the problem of stent segmentation is somehow very close to the problem of blood vessel segmentation (see [12] for a review on the topic which concerns traditional approaches). Notably, some multi-scale filters have met success in the task of blood vessel segmentation [6] [14], these methods will be used for comparisons in the result section. During the last years, deep learning approaches are performing state of the art results in the context of supervised segmentation. Results using this technique are also available for 3D vessel segmentation [13], and could potentially be adapted to 3D stent segmentation. However, this goes out of the scope of this article which deals with unsupervised segmentation.

Finally, a point should be made about a very close and active research topic which is that of Metal Artifact Reduction (MAR) [17] [19]. MAR focuses on improving the quality of images corrupted by metallic artifacts. As opposed to this litterature we do not aim at reconstructing the original image with reduced artifacts: the purpose of our article is to precisely perform segmentation in presence of artifacts. However, since many MAR approaches first rely on a segmentation of the metallic elements in the image, the work presented in this article contributes to the improvement of MAR techniques. This will be explored in the last section.

\section{Fine AND UNSUPERVISED STENT SEGMENTATION}

This section step-by-step describes the whole segmentation process to segment stents in X-ray scans corrupted by artifacts. In this unsupervised segmentation problem two classes are of interest, we call them the stent class and the rest class. Figure 2 summarizes the whole process.

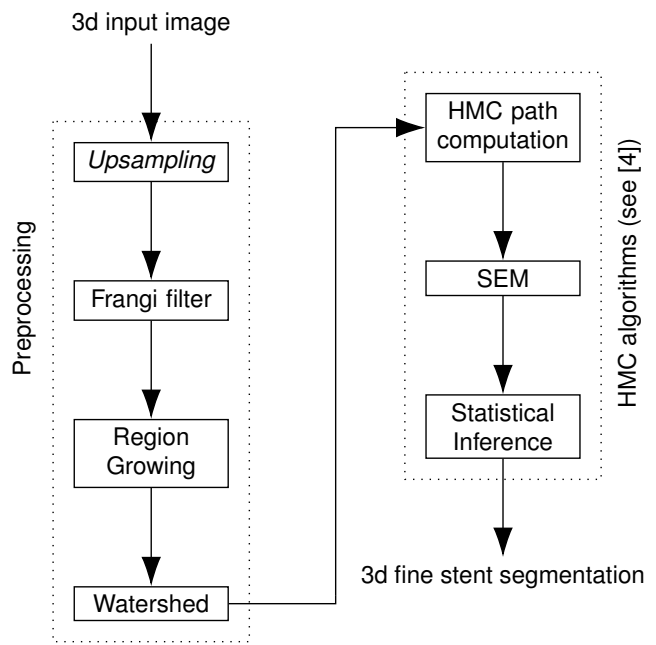

Fig. 2: Diagram of the fine stent segmentation step.

\section{A. Preprocessing for a coarse segmentation}

We present an approach for a first coarse segmentation of the stent which serves as an initialization of a probabilistic fine segmentation method. This algorithm corresponds to the Preprocessing section of Figure 2.

The Frangi filter [6] is first performed and we keep only voxels whose probability to belong to a tubular structure is above a certain threshold (dependent on the nature of the image we want to segment). A region-growing algorithm is performed in $3 \mathrm{D}$ to select only the biggest connected component. A watershed algorithm [8] is performed on every slice to separate connected components that should be disjoint but still appear stuck together because of the artefacts.

Remark: A step of upsampling is needed for images of very low resolution such as CT scans (see, e.g., Figure 1b). The idea is that the stent parts on the image should be increased up to a certain width (we used 10 pixels in our applications) so that the successive filters used for segmentation show responses.

Remark: The Frangi filter is the most important part of the Preprocessing pipeline. The subsequent HMC algorithms can be seen as a statistical refinement of the Frangi filter response, increasing the robustness of the latter.

\section{B. Fine segmentation with Hidden Markov Chains}

A fine segmentation step is needed to recover the smooth and regular stent shape. Moreover this refinement step should not require any prior knowledge about the stent structure due to the heterogeneity of the data. The Hidden Markov Chains (HMC) are an intuitive way to introduce dependence between neighboring voxels to get the desired processing on the stent [1]. The novelty of our approach also lies in the choice of the HMC path and in the noise model. 


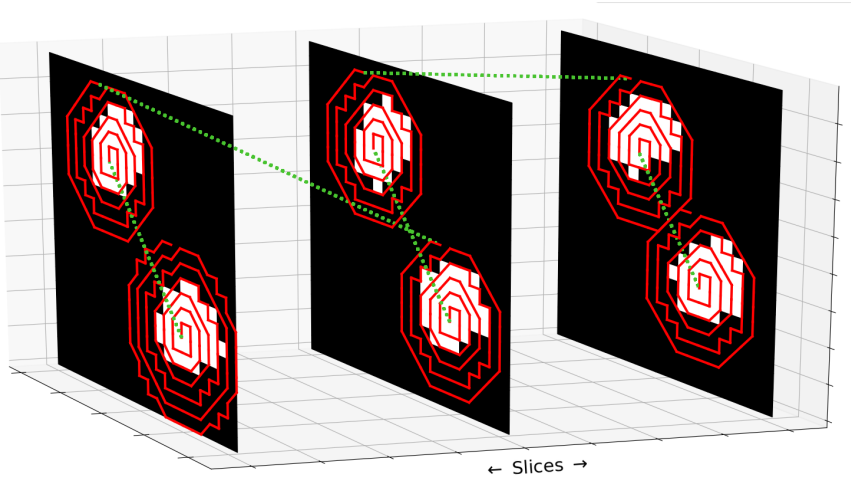

Fig. 3: The HMC path illustrated on 3 successive slices.

1) The HMC path: When using a HMC to segment an image, one must carefully consider its path through the image, i.e. the 3D image data must be ordered into a $1 \mathrm{D}$ sequence. A classical way to do this for the whole data cube is to use a 3D Hilbert-Peano scan, as applied in [2].

However, we propose another kind of path to handle the artifacts. Each connected component (of the stent class from the coarse segmentation) of each slice is visited using a snail path (snail paths are described in [18]). The sense of rotation is alternated between the snail paths. This way, physical continuity in the path is simulated, gathering all the stent parts in a 1D sequence which allows efficient computations. Such a path is particularly suited to get a smoothed and regular surface for tubular structures [4]. Moreover, in the HMC model one has to learn the transition probabilities between the classes. A small morphologic dilation is then performed over the result of the coarse segmentation for the snail paths to be a little bigger than the connected components isolated during the coarse segmentation. Thus, we also make sure that all the voxels belonging to the stent class are part of the sequence of pixels to classify.

Figure 3 illustrates our choice for the path of the Markov chain through the slices of our data cubes.

2) The HMC model: We follow the statistical approach developped in [4]. We refer the reader to the latter article for all the details about the statistical modeling. In our work, the hidden variables take the value of stent or rest forming the segmentation image. The observed variables represent the pixel values of the observed scan. The unsupervised parameter estimation is carried with the Stochastic Expectation Minimization (SEM) algorithm [3].

3) Improving the noise model to handle strong artefacts: Classically, such as in [4], the noise model in an HMC is chosen to be a mixture of Gaussian laws (MoG). Similarly to [15], we propose to work with a noise model involving mixture of exponential laws (MoE) to improve the results. Indeed, the "all-or-nothing" behaviour that we get by using the exponential law seems particularly suited to handle the strongest artifacts.
The exponential probability density function is given by:

$$
f(x ; \lambda, \delta)= \begin{cases}\lambda \exp (-\lambda(x-\delta)) & x>\delta \\ 0 & x \leq \delta\end{cases}
$$

where $(\lambda, \delta) \in\left(\mathbb{R}^{+*}, \mathbb{R}\right)$ are the new parameters to estimate for each class in the SEM procedure.

Moreover, a likelihood score comparison was conducted and showed that exponential laws fitted better the empirical distribution (obtained after the coarse segmentation) for the stent class and the rest class than the Gaussian laws.

\section{RESUlTS}

\section{A. Unsupervised stent segmentation results on $C T$ and $m C T$}

The task of unsupervised segmentation in X-ray scans is a complex problem. Notably, artifact and calcification pixels are close to the stent pixels both in intensity, geometry and localization, which tends to produce stent false-positive pixels with the common algorithms mentionned above. We begin by illustrating segmentations by our fine segmentation method, the RORPO filter ${ }^{2}$ segmentation [14], the manual thresholding segmentation and the Frangi filter ${ }^{2}$ segmentation [6]. The last two approaches are still the most common approach for metallic stent segmentation. Note that all methods operate in $3 \mathrm{D}$ but $2 \mathrm{D}$ slices are first presented for a better visual assessment of the results. Figure 4 illustrates the unsupervised segmentation experiments. Figure 5 illustrates some segmented 3D meshes of broken stents.

The absence of ground truth forces us to qualitative assessment of the segmentations. Our algorithm is the most likely to offer a precise refinement over the stent pixels as well as avoiding false positive resulting from calcifications or artefacts.

It is notable that the results from the three methods we compare our method with depend on a final step of manual thresholding. On the contrary, our algorithm does not need such manual intervention since the statistical refinement is fully automatic, avoiding a time consuming step for the expert which is also subject to the operator subjectivity.

\section{B. Application to MAR}

As stated in the introduction, we can apply our new metal segmentation method to improve Metal Artifact Reduction (MAR) procedures. Beam Hardening Correction (BHC) is built upon the Linear Interpolation (LI) technique and these approaches form are the most classical for MAR [17] [19]. This section shows, through Figure 6, on various CT scans, that we are able to restaure images with reduced artifacts using our fine statistical segmentation followed by BHC. Results are compared to a manual segmentation, to a segmentation based on Frangi filter and to a RORPO-based segmentation, all three followed by BHC. Such results highlight the importance of the metallic segmentation step in MAR problems.

\footnotetext{
2 This filter response over the data cube is manually segmented afterwards, with a global threshold.
} 


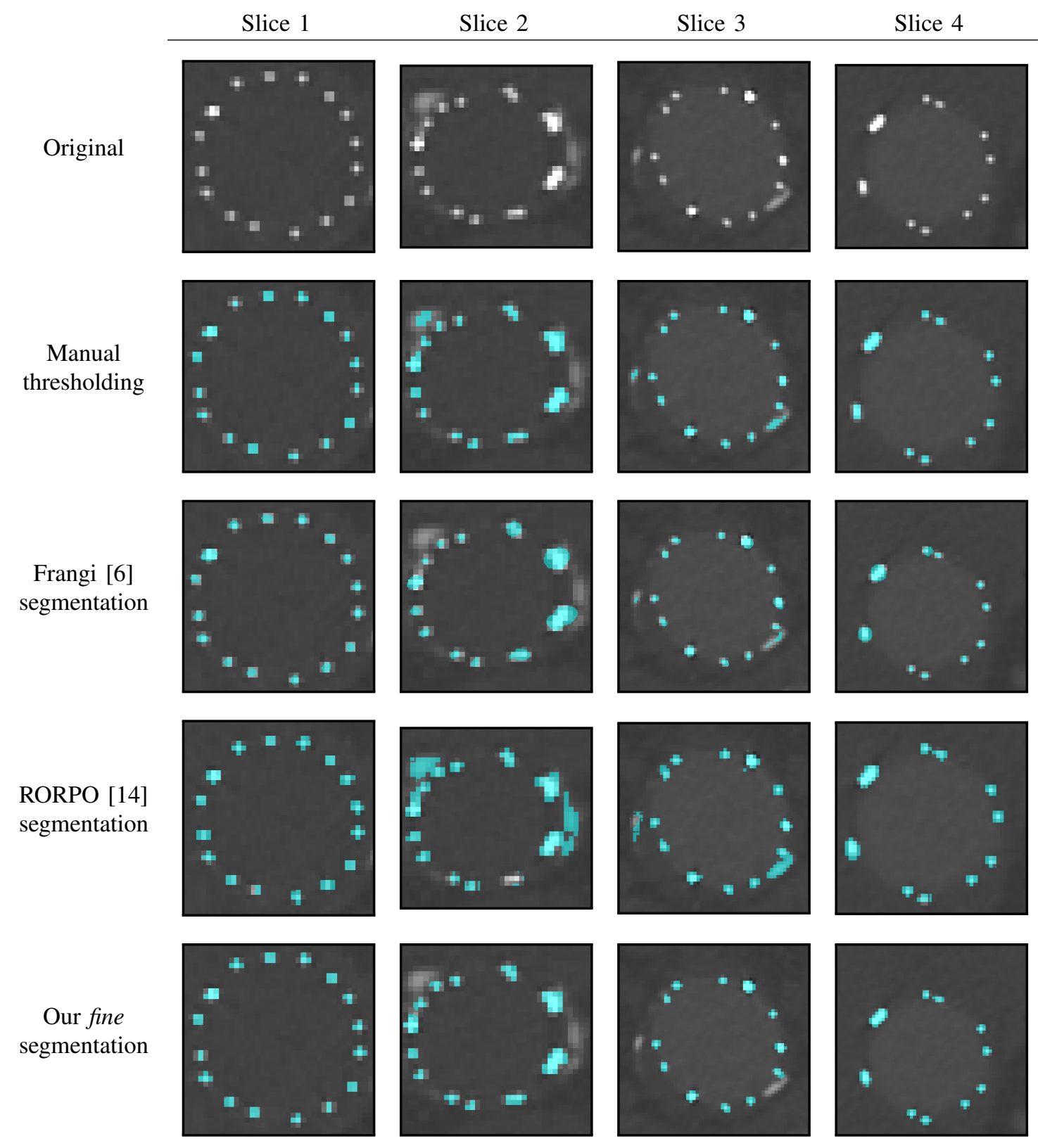

Fig. 4: Unsupervised stent segmentation of stents in different scans, with different methods. The scales used in the Frangi filter are $\{3,6,9\}$. The scales used in the RORPO filter are $\{100,150,200\}$.
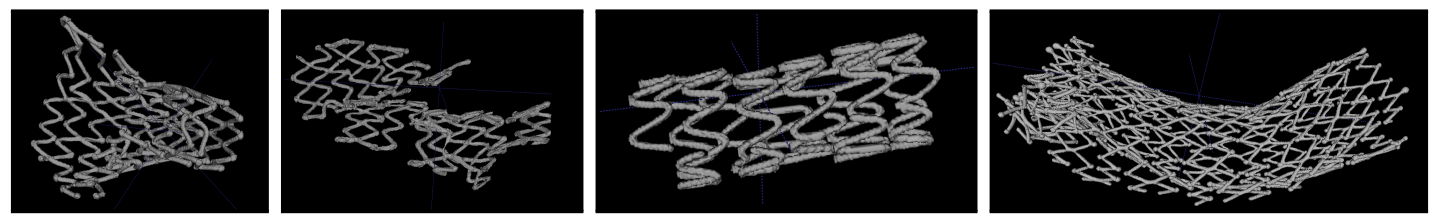

Fig. 5: 3D view of segmented mCT of stents from the database. 
Slice 5

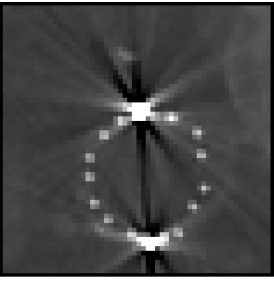

Manual thresholding

$+\mathrm{BHC}$

Original

slices

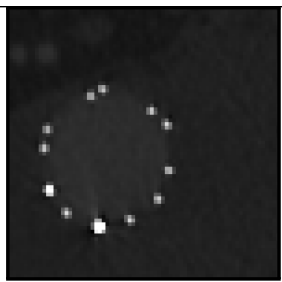

Slice 7

$$
\begin{aligned}
& \text { RORPO [14] } \\
& \text { segmentation } \\
& + \text { BHC }
\end{aligned}
$$

segmentation

$+\mathrm{BHC}$

Our fine

segmentation

$+\mathrm{BHC}$
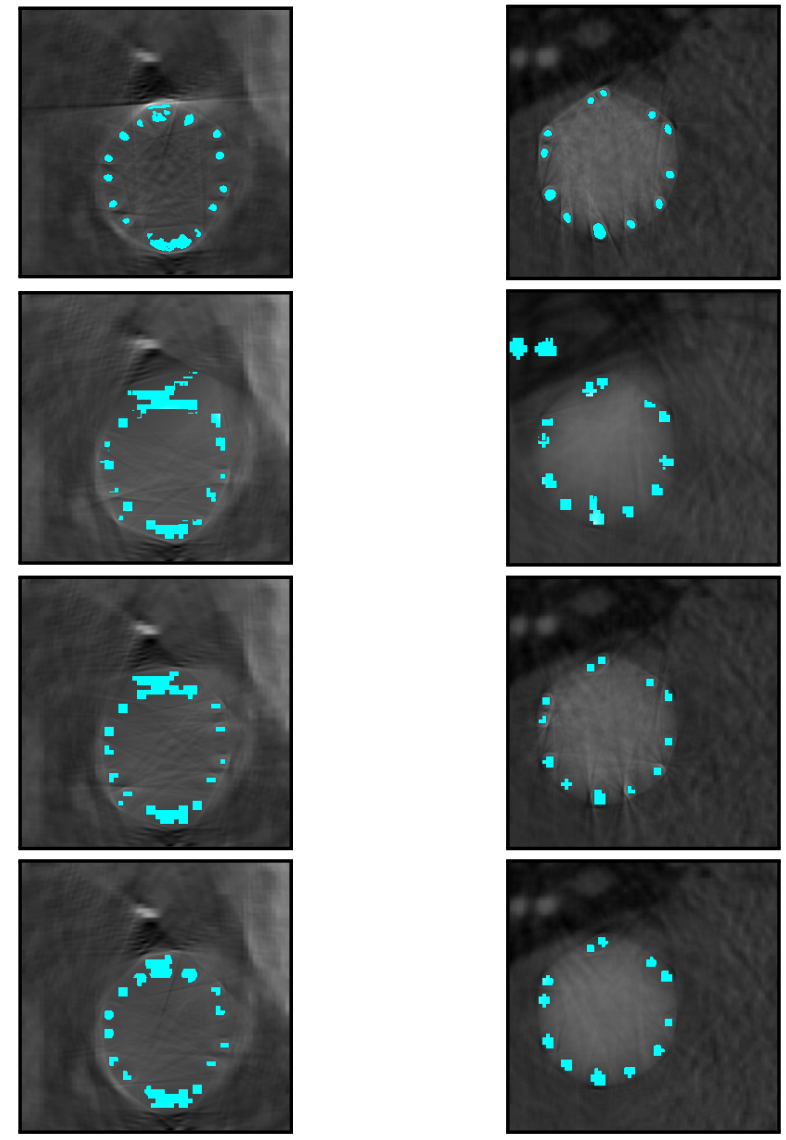
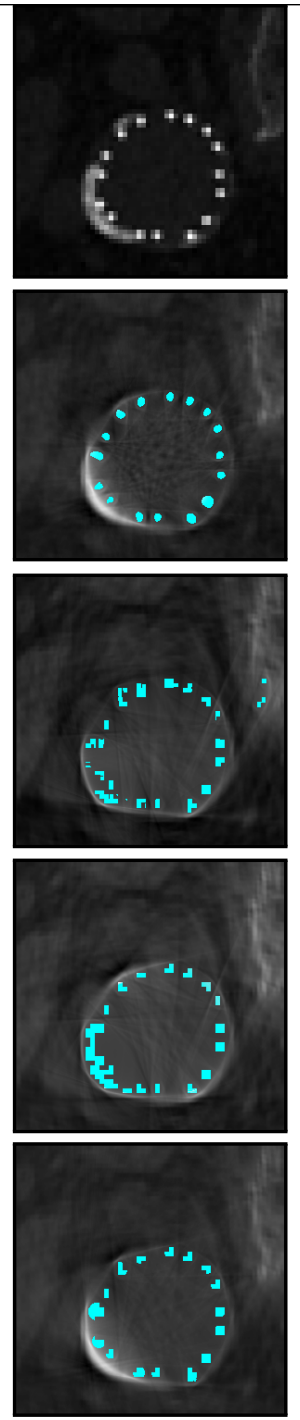

Fig. 6: MAR with the BHC algorithm when using different metal segmentation techniques. The last row, which is the one including our segmentation technique, shows restaured images less affected by the artifacts. This can be seen especially in the center region of the stent. Artifacts may be hard to see on the original slices because of the image dynamic range. The scales used in the Frangi filter are $\{3,6,9\}$. The scales used in the RORPO filter are $\{100,150,200\}$.

\section{Effects of the mixture of exponential}

This last subsection illustrates the refinement introduced by using a noise model involving a mixture of exponential laws, which better models the nature of the artifacts in the images. Indeed, as depicted in Figure 7 on two cases with very strong artifacts, the recovered stent structure is much thinner and much more circular as the original metallic structure of the stent.

\section{CONCLUSION}

In this article we developped an automatic and unsupervised segmentation technique for metallic stents in complex and corrupted images. It is suitable in $\mathrm{CT}$ and $\mathrm{mCT}$ images. Using a dedicated statistical construction, our approach exhibits a better robustness to artifacts and to calcifications and constitutes an improvement over classical segmentation techniques of noisy tubular structures. We proved the interest of our approach for the problem of metallic artifact reduction.

Further work might consider complexifying the noise model to take into account the correlated noise, which has been proven possible within the Hidden Markov Models family [7].

\section{ACKNOWLEDGMENT}

The authors would like to thank S. Kuntz, M. D. Kutyna and R. Virmani from CVPath Institute Inc. (Gaithersburg, Maryland, USA) for their collaboration to this work and for the acquisition of the $\mathrm{mCT}$ images. 


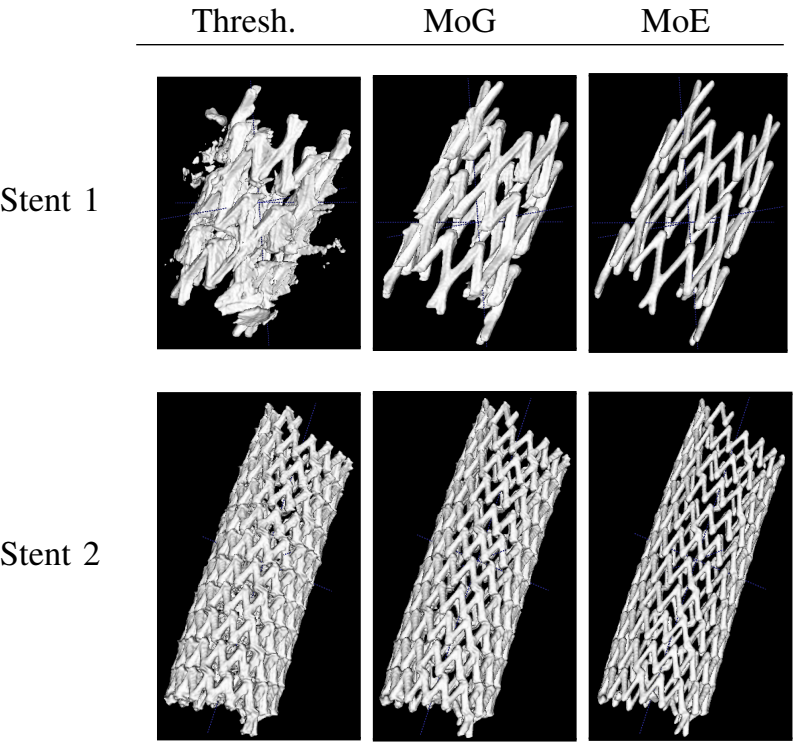

Fig. 7: 3D segmentation of two stents via manual thresholding (Thresh.), MoG approach and MoE approach.

\section{REFERENCES}

[1] L. E. Baum et al. "A maximization technique occurring in the statistical analysis of probabilistic functions of Markov chains". In: The annals of mathematical statistics 41.1 (1970), pp. 164-171.

[2] S. Bricq et al. "MS lesion segmentation based on hidden Markov chains". In: Grand Challenge Work.: Mult. Scler. Lesion Segm. Challenge (2008), pp. 1-9.

[3] G. Celeux and J. Diebolt. "A stochastic approximation type EM algorithm for the mixture problem". In: Stochastics: An International Journal of Probability and Stochastic Processes 41.1-2 (1992), pp. 119-134.

[4] J.-B. Courbot et al. "Vertebra segmentation based on two-step refinement". In: Journal of computational surgery 4.1 (2016), p. 1.

[5] S. Demirci et al. "3d stent recovery from one x-ray projection". In: International Conference on Medical Image Computing and Computer-Assisted Intervention. Springer. 2011, pp. 178-185.

[6] A. F. Frangi et al. "Multiscale vessel enhancement filtering". In: International Conference on Medical Image Computing and Computer-Assisted Intervention. Springer. 1998, pp. 130-137.

[7] I. Gorynin et al. "Assessing the segmentation performance of pairwise and triplet Markov models". In: Signal Processing 145 (2018), pp. 183-192.

[8] M. Haidekker. Advanced biomedical image analysis. John Wiley \& Sons, 2011.

[9] A. Klein et al. "Automatic segmentation of the wire frame of stent grafts from CT data". In: Medical image analysis 16.1 (2012), pp. 127-139.
[10] G. Langs et al. "Learning deformation and structure simultaneously: In situ endograft deformation analysis". In: Medical image analysis 15.1 (2011), pp. 12-21.

[11] A. Lejay et al. "Explanted vascular and endovascular graft analysis: where do we stand and what should we do?" In: European Journal of Vascular and Endovascular Surgery (2018).

[12] D. Lesage et al. "A review of 3D vessel lumen segmentation techniques: Models, features and extraction schemes". In: Medical image analysis 13.6 (2009), pp. 819-845.

[13] M. Livne et al. "A U-Net deep learning framework for high performance vessel segmentation in patients with cerebrovascular disease". In: Frontiers in neuroscience 13 (2019).

[14] O. Merveille et al. "Tubular Structure Filtering by Ranking Orientation Responses of Path Operators". In: European Conference on Computer Vision (ECCV). Vol. 8690. Springer, 2014, pp. 203-218.

[15] E. Monfrini and W. Pieczynski. "5-Estimation de mélanges généralisés dans les arbres de Markov cachés, application à la segmentation des images de cartons d'orgue de barbarie". In: (2005).

[16] D. Perrin et al. "Patient-specific simulation of endovascular repair surgery with tortuous aneurysms requiring flexible stent-grafts". In: Journal of the mechanical behavior of biomedical materials 63 (2016), pp. 86-99.

[17] J. M. Verburg and J. Seco. "CT metal artifact reduction method correcting for beam hardening and missing projections". In: Physics in Medicine \& Biology 57.9 (2012), p. 2803.

[18] M. Yahiaoui et al. "Implementation of unsupervised statistical methods for low-quality iris segmentation". In: 2014 Tenth International Conference on SignalImage Technology and Internet-Based Systems. IEEE. 2014, pp. 566-573.

[19] Y. Zhang and Hengyong Yu. "Convolutional neural network based metal artifact reduction in x-ray computed tomography". In: IEEE transactions on medical imaging 37.6 (2018), pp. 1370-1381. 